\title{
Author Index Vol. 64 (suppl 1), 1995
}

Alessandrini, F. 72,228 Andou,Y. 32,110,164,249

Babighian, S. 56,67,72,134

Bauman, G. 118

Benati,A. 67,72,228

BergloffJ. 233

Berlucchi, S. 56

Bissonette, DJ. 87

Bonomi, L. 67, 72

Bortolazzi, E. 134

Bricolo, A. 42, 56, 67,72,134,228

Chang, J.W. 239 Chang, Y. 258 Chen, K.Y. 258 Cheng, S.S. 258 Cho,J. 239 Chung, S.S. 239 Chung, W.Y. 19,258 Ciricillo,S. 118 Copcutt,B.G. 172

Dufour,H. 182

Edwards, M. 118 Ertl,A. 80

Fang,B. 147 Feichtinger, H. 11 Ferrari, S. 42 
Flickinger, J.C. 87 Foroni, R. 42, 56,67,72,134, 228 Forster,rSS[.C. 155 Friehs,G.M. 11,209 Fukuoka,S. 98

GanzJ.C. 1,3

Gerosa, M.A. 42,56,67,72,134,228

Ghimenton, C. 42

Giri, M.G. 42, 56, 67, 72,134,228

Gould, K.L. 147

Grimm, P. 172

Guo,W.Y. 19,258

Gutin,P. 118

Ha,C.-S. 147 Hampshire, A. 155 Hayashi,S. 249 Hirato,J. 32

Hirato,M. 32,110,164,249 Horikoshi,S. 249

Ide,M. 126

Inoue, H.K. 32,110,164,249 Ishihara,J. 249 Iuzzolino, P. 42

Jacques, D.B. 172 Jimbo, M. 126 
Kemeny.A.A. 155 Kim.CJ. 202 Kim,D.I. 239 Kitz,K. 80 Kohga,H. 110 Kondziolka, D.S. 87 Kramer, L. A. 147 Kurihara, H. 110 Kwon,Y. 222

Lamki,LX. 147 Langmann, G. 233 Larson, D. 118 Leber, K.A. 233 Lee,L.S. 19,258

Levrier.O. 193 Lindquist, C. 126 Liu,R.S. 19,258 Lunsford, D.L. 8 LuzzatLR. 42

Manera,L. 182 Marchini, G. 56, 67, 72,134 McDermott,M. 118 Menapace, R. 80 Mokry,M. 233 Mullani,N. 147

Nagashima, K. 98 Nakagawara, J. 98 Nakamura,J. 98 Nakamura,M. 32,164

Nicolato, A. 42, 56,67,72,134,228

Ogunrinde, O.K. 87 Ohye,C. 110,164,249 Ojakangas, C.L. 209 Ott.E. 209

Pachatz,P. 209

Pan,D.H.C. 19,258

Park,Y.G. 239

Pasoli, A. 42, 56,67,72,134,228 
Pasqualin, A. 134

Pendl,G. 11,209,233

Peragut,J.C. 182,193

Petti, P. 118

Piovan, E. 42, 56,67, 72,134,228

Porcheron, D. 182,193

Posewitz, A.E. 172

Rand,R.W. 172 Regis, H. 193 Regis, J. 182,193 Rey.M. 193

Samson, Y. 193 Schrottner, O. 11,209,233 Sedan, R. 182,193 Seo,Y. 98 Shiau,C.Y.

19,258 Shibazaki, T. 32,110,164,249 Smith, V. 118 Sneed,P. 118 Steiner.L. 126

Suematsu, K. 98

Takanashi,M. 98 Tomazzoli, L. 67,72

Verhey,L. 118 Vermeulen, S.S. 172

Walsh, J.W. 147 Walton, L. 155 Wara,W. 118 Whang, C.J. 202,222

Yamamoto, M. 126 Yeh, S.H. 258 Young, R.F. 172

Zama, A. 249

Zampieri, P.G. 42, 56, 67,72,134,228

Zeck,O.F. 147

Zehetmayer, M. 80

Author Index 
